Check for updates

Cite this: Mater. Chem. Front., $2019,3,650$

Received 21st January 2019 ,

Accepted 13th February 2019

DOI: $10.1039 / c 9 q m 00036 d$

rsc.li/frontiers-materials

\section{Biocompatible small organic molecule phototheranostics for NIR-II fluorescence/ photoacoustic imaging and simultaneous photodynamic/photothermal combination therapy $\dagger$}

\author{
Qi Wang, ${ }^{a}$ Bing Xia, ${ }^{a}$ Jingzeng $\mathrm{Xu}{ }^{\mathrm{a}}$ Xinrui Niu, ${ }^{\mathrm{a}}$ Jie Cai, ${ }^{\mathrm{a}}$ Qingming Shen, ${ }^{\mathrm{a}}$ \\ Wenjun Wang, ${ }^{\mathrm{b}}$ Wei Huang ${ }^{\mathrm{ac}}$ and Quli Fan (D) *ac
}

\begin{abstract}
The integrated functionalities of multiple diagnostic and therapeutic modalities in a single phototheranostic nanoplatform have been validated as a significant breakthrough toward cancer theranostics. Nevertheless, most reported nanoplatforms are constructed by using complex components through difficult fabrication processes, and often need different excitation wavelengths. Herein, a novel small-molecule dye, DPP-BDT, with absorption in the NIR-I window and fluorescence emission in the NIR-II region was designed and synthesized, and it presented excellent photodynamic and photothermal performance. Based on single component DPP-BDT, multifunctional phototheranostics were rationally and successfully constructed. From in vitro and in vivo experiments, such nanotheranostics showed excellent tumor destruction abilities under a single wavelength of laser irradiation, benefiting from the NIR-II fluorescence/photoacoustic dual-modal imaging guided photodynamic/photothermal combination therapy.
\end{abstract}

\section{Introduction}

With the development of optical technology, phototheranostics, which offer simultaneous light-induced diagnostics, imaging, and therapy, have attracted increasing attention in the field of cancer theranostics. ${ }^{1}$ To date, although remarkable progress in phototheranostics has been achieved during the past few decades, most of the current systems only involve single-mode imaging guided monotherapy, which leads to limited diagnostic accuracy and therapeutic outcome. ${ }^{2}$ As one of the most notable approaches to overcoming these drawbacks, multimodal imaging guided combination therapy, which merges the superiorities of multiple imaging and treatment modalities, provides a simple

\footnotetext{
${ }^{a}$ Key Laboratory for Organic Electronics and Information Displays \& Jiangsu Key Laboratory for Biosensors, Institute of Advanced Materials (IAM), Jiangsu National Synergetic Innovation Center for Advanced Materials (SICAM), Nanjing University of Posts \& Telecommunications, Nanjing 210023, China. E-mail:iamqlfan@njupt.edu.cn

${ }^{b}$ Key Lab of Optical Communication Science and Technology of Shandong Province \& School of Physics Science and Information Engineering, Liaocheng University, Liaocheng 252059, China

${ }^{c}$ Shaanxi Institute of Flexible Electronics (SIFE), Northwestern Polytechnical University (NPU), 127 West Youyi Road, Xi'an 710072, China

$\dagger$ Electronic supplementary information (ESI) available: Experimental details, NMR spectra, etc. See DOI: 10.1039/c9qm00036d
}

and efficient approach to enhance the accuracy of diagnosis and improve the efficacy of therapy. Recently, fluorescence imaging in the second near infrared window (NIR-II, 1000-1700 nm) and photoacoustic (PA) imaging, as newly emerged non-invasive optical imaging modalities, have attracted tremendous interest for tumor diagnosis. ${ }^{3}$ NIR-II fluorescence imaging can offer higher signal-to-noise ratio, better imaging qualities and deeper tissue penetration than those observed in the NIR-I window (650-900 $\mathrm{nm})$ due to the diminished photon scattering and tissue auto-fluorescence. ${ }^{4}$ PA imaging, which integrates the merits of ultrasound imaging and optical imaging, has the ability to describe deep tumor margins with microscopic spatial resolution. ${ }^{5}$ Despite remarkable progress in NIR-II fluorescence imaging and PA imaging systems, comprehensive and accurate cancer diagnosis information cannot be effectively obtained by only a single imaging modality. ${ }^{3 a, 6}$ Therefore, the strategy of integrated NIR-II fluorescence imaging and PA imaging holds great promise to increase diagnostic accuracy. ${ }^{7}$

As promising alternatives to traditional tumor treatments, photodynamic therapy (PDT) and photothermal therapy (PTT), as two major categories of phototherapy, are emerging as noninvasive, harmless and effective therapeutic modalities. ${ }^{8}$ PDT usually employs photosensitizers to kill cancer cells through highly cytotoxic reactive oxygen species (ROS, typically singlet oxygen ${ }^{1} \mathrm{O}_{2}$ ). ${ }^{9}$ PTT utilizes photothermal agents to convert light 
energy into heat, resulting in thermal ablation of cancer cells. ${ }^{10}$ Nevertheless, photosensitizers or photothermal agents often decay gradually under light irradiation. ${ }^{11}$ Moreover, the efficiency of PDT and PTT is seriously inhibited by the hypoxia microenvironment around the tumor and the acquired thermal-resistance properties of residual cancer cells, respectively, leading to unsatisfactory therapeutic outcome by either PDT or PTT. ${ }^{12}$ What is exciting is that the combination of PDT and PTT is considered as a groundbreaking approach to address these issues and enhance the therapeutic outcome. ${ }^{13}$

To date, a series of inorganic nanomaterials have been developed for NIR-II fluorescence/PA dual-modal imaging or imaging-guided therapy. ${ }^{4 b, 7 b, 14}$ However, the potential cytotoxicity of inorganic materials largely restricts their practical application. Meanwhile, these nanomaterials are usually constructed by using multicomponent composites through complicated preparation processes. ${ }^{2 a}$ Additionally, different excitation wavelengths are often required to achieve their respective functions, which causes systematic complexity and increased treatment times. ${ }^{15}$ Compared with inorganic materials, organic dyes, especially small organic molecules, offer irreplaceable advantages in clinical practice, such as outstanding biocompatibility, and fine-tuned optical properties and chemical structures. ${ }^{16}$ In 2017, Cheng and colleagues synthesized a benzobisthiadiazole-based small-molecule NIR-II fluorophore for dual-modal NIR-II fluorescence and PA imaging. However, the therapeutic functionality of this NIR-II fluorophore was not reported. ${ }^{7 a}$ To further expand and enrich the theranostic study of cancer, intelligently devised single-component small organic molecule based phototheranostics that combine the functions of NIR-II fluorescence imaging, PA imaging, PDT and PTT, triggered by a single wavelength laser, are urgently desired.

Herein, a novel small-molecule dye, DPP-BDT, with absorption in the NIR-I window and fluorescence emission in the NIR-II region was synthesized. Multifunctional phototheranostic nanoparticles, DPP-BDT NPs, were then obtained by encapsulating the DPP-BDT dye with amphiphilic DSPE-mPEG5000, and showed good water-solubility, outstanding biocompatibility, and high photostability. Moreover, such DPP-BDT NPs could generate toxic ROS and hyperthermia simultaneously under a single NIR laser irradiation. In vitro and in vivo assays confirmed that these nanoparticles could realize remarkable antitumor efficacy under single wavelength laser irradiation, benefiting from the NIR-II fluorescence/PA dual-modal imaging guided PTT/PDT combination therapy (Scheme 1).

\section{Results and discussion}

Diketopyrrolopyrrole (DPP) as a strong electron acceptor (A) was conjugated with an electron donor (D), benzodithiophene (BDT), through a palladium-catalyzed Stille coupling reaction to form an A-D-A type of small-molecule, NIR-II dye DPP-BDT, which was confirmed by ${ }^{1} \mathrm{H}$ NMR, ${ }^{13} \mathrm{C}$ NMR, and LR-ESI-MS (Scheme S1, ESI $\dagger$ ). Amphiphilic DSPE-mPEG5000 was employed to encapsulate the hydrophobic DPP-BDT to provide water-soluble phototheranostic nanoparticles, DPP-BDT NPs. The transmission

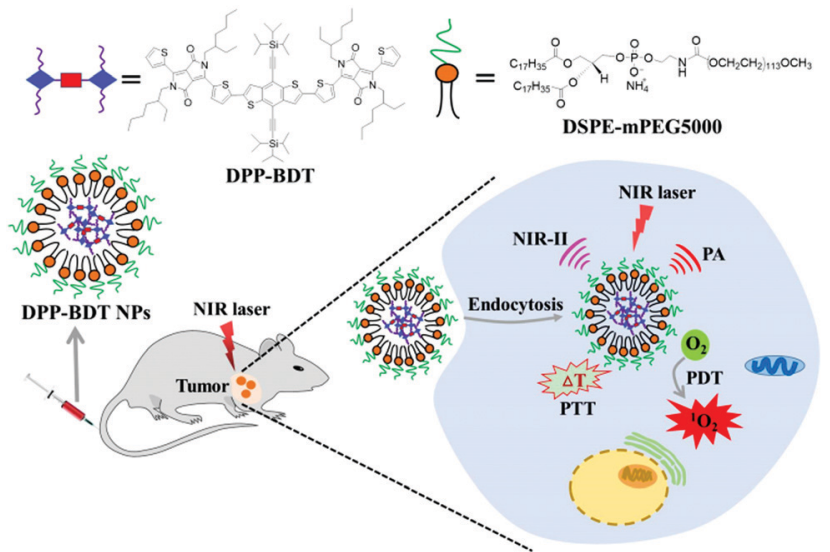

Scheme 1 Schematic illustration of small organic molecule phototheranostics for NIR-II fluorescence/PA imaging and simultaneous PDT/PTT combination therapy.

electron microscopy (TEM) image of the DPP-BDT NPs displayed a spherical morphology with diameters of approximately $90 \mathrm{~nm}$, which was close to the result from dynamic light scattering (DLS, Fig. 1a). The as-prepared DPP-BDT NPs displayed no noticeable size change over 16 days, indicating that the NPs were relatively stable (Fig. S4, ESI $\dagger$ ). From the UV-vis-NIR spectrum (Fig. 1b), the DPP-BDT NPs exhibited a maximum absorption peak at $625 \mathrm{~nm}$ and two shoulder peaks at $667 \mathrm{~nm}$ and $750 \mathrm{~nm}$. The molar absorption coefficient of the DPP-BDT NPs at $660 \mathrm{~nm}$ was $3.35 \times$ $10^{4} \mathrm{M}^{-1} \mathrm{~cm}^{-1}$ (Fig. S5, ESI $\dagger$ ). Compared with commercial dye methylene blue (MB), the DPP-BDT NPs displayed outstanding photostability even after $90 \mathrm{~min}$ of continuous $660 \mathrm{~nm}$ laser irradiation (Fig. S6, ESI $\dagger$ ). The PA imaging performance of the DPP-BDT NPs in vitro at different concentrations under excitation at $660 \mathrm{~nm}$ was recorded (Fig. 1c). The PA signal values produced by the DPP-BDT NPs were linearly proportional to the concentration of the NPs, and the brightness of the PA images was also gradually enhanced, confirming that the DPP-BDT NPs can act as a superb
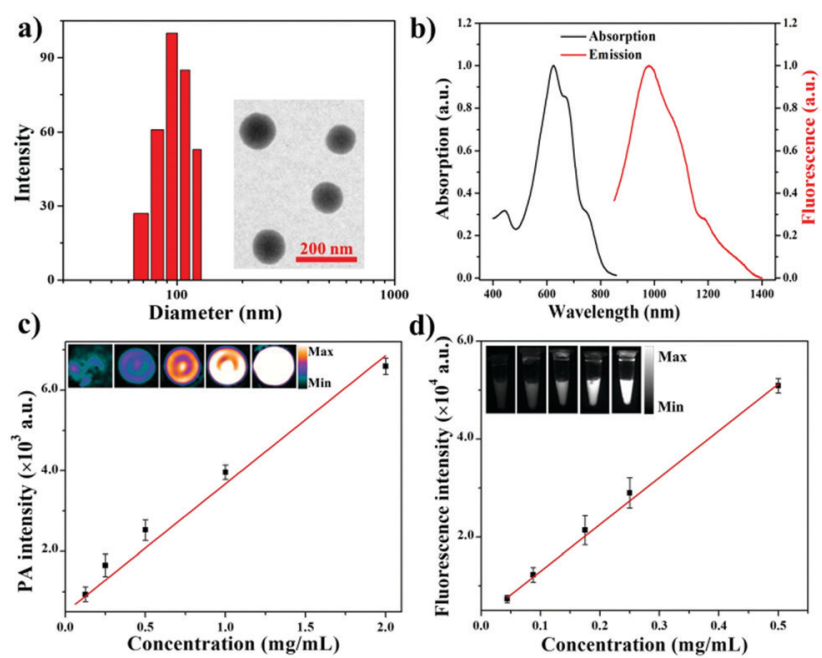

Fig. 1 (a) DLS results (polydispersity: 0.10) and TEM data (inset) of the DPP-BDT NPs. (b) Absorbance and fluorescence spectra of the DPP-BDT NPs. (c) Fluorescence mapping and (d) PA mapping of the DPP-BDT NPs. 
PA imaging contrast agent. Moreover, the DPP-BDT NPs presented excellent fluorescence emission in the NIR-II region with a maximum peak at $980 \mathrm{~nm}$ (Fig. 1b). Then, the NIR-II fluorescence imaging performance of the DPP-BDT NPs was tested. The changes in NIR-II fluorescence signal of the DPP-BDT NPs at different concentrations were in good agreement with the PA imaging results, presenting a linear relationship with concentration. The NIR-II fluorescence quantum yield (QY) of the DPP-BDT NPs was determined to be $\mathbf{0 . 5 2} \%$. More interestingly, after covering a polyethylene tube with chicken-breast tissue of varying thickness, the NIR-II imaging of the DPP-BDT NPs reached a depth of $9 \mathrm{~mm}$, demonstrating that the DPP-BDT NPs have excellent advantages for NIR-II fluorescence imaging (Fig. S7, ESI $\dagger$ ).

In view of the excellent optical properties of the DPP-BDT NPs, the capacity of the DPP-BDT NPs for PTT was systematically investigated. In Fig. 2a, an insignificant temperature increase of water was observed upon $660 \mathrm{~nm}$ laser irradiation for $10 \mathrm{~min}$ $\left(0.75 \mathrm{~W} \mathrm{~cm}^{-2}\right)$. However, under identical laser conditions, hyperthermia of the DPP-BDT NP solution can be effectively obtained with an increase in the concentration of the NPs, which was confirmed by the color changes of the corresponding infrared (IR) thermal images (Fig. S8, ESI $\dagger$ ). The temperature increase of the NP solution is also proportional to the laser power density (Fig. 2b). Moreover, no obvious changes in temperature elevation were observed after five cycles of $660 \mathrm{~nm}$ laser irradiation, displaying that the DPP-BDT NPs have excellent photothermal stability (Fig. S9, ESI $\dagger$ ). The photothermal conversion efficiency of the DPP-BDT NPs was calculated to be about 23.0\% (Fig. S10, ESI $\dagger$ ). The capability of these NPs to produce ROS upon laser irradiation was further investigated by using 1,3diphenylisobenzofuran as a probe (DPBF, a typical ${ }^{1} \mathrm{O}_{2}$ indicator). In Fig. 2c, a significant decrease of DPBF absorbance in the NP solution was observed with the increase of the laser power
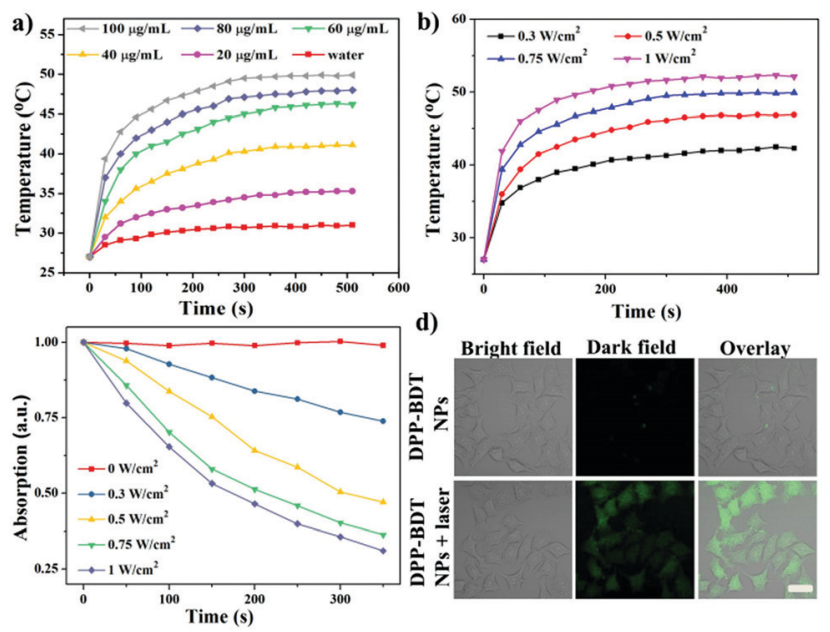

Fig. 2 Photothermal curves of the DPP-BDT NPs (a) at various concentrations $\left(660 \mathrm{~nm}, 0.75 \mathrm{~W} \mathrm{~cm}^{-2}\right.$ ) and (b) with different power densities ([DPP-BT] = $100 \mu \mathrm{g} \mathrm{mL}^{-1}$ ). (c) Absorption change of DPBF over time at various laser power densities $(660 \mathrm{~nm}$ ). (d) Intracellular ROS generation of HeLa cells treated with the DPP-BDT NPs with or without irradiation $(660 \mathrm{~nm}$, $\left.0.75 \mathrm{~W} \mathrm{~cm}^{-2}, 10 \mathrm{~min}\right)$. Scale bars: $20 \mu \mathrm{m}$. density. In particular, when the NPs were exposed to a $660 \mathrm{~nm}$ laser of $1.0 \mathrm{~W} \mathrm{~cm}^{-2}$, the relative absorbance decreased by $70.6 \%$ within $350 \mathrm{~s}$, suggesting the excellent ${ }^{1} \mathrm{O}_{2}$ generation capability of the NPs. The ${ }^{1} \mathrm{O}_{2}$ quantum yield of the DPP-BDT NPs was calculated to be about $49.3 \%$ (Fig. S11, ESI $\dagger$ ). Non-fluorescent $2^{\prime}, 7^{\prime}$-dichlorofluorescein diacetate (DCFH-DA), which can be rapidly oxidized by ROS to green fluorescent $2^{\prime}, 7^{\prime}$-dichlorofluorescein (DCF), was used as an indicator to further assess the ROS generation capability of these NPs in living cells. In Fig. 2d, the HeLa cells treated with NPs and DCFH-DA displayed strong green fluorescence upon irradiation with a $660 \mathrm{~nm}$ laser. Meanwhile, insignificant fluorescence of the groups without laser irradiation was found, suggesting that the DPP-BDT NPs can intracellularly produce ROS upon laser irradiation. All of these results indicated that the DPP-BDT NPs could serve as excellent candidates for NIR-II fluorescence/PA dual-modal imaging, as well as PTT/PDT combination therapy under single laser irradiation.

Before applying DPP-BDT NPs as multifunctional phototheranostics in vivo, the biocompatibility of the DPP-BDT NPs was estimated using an MTT assay against NIH-3T3 normal cells. Negligible changes in cell viability were discovered with concentrations of the DPP-BDT NPs ranging from 0 to $25 \mu \mathrm{g} \mathrm{mL}{ }^{-1}$, indicating outstanding biocompatibility of these NPs (Fig. 3a). Next, the in vitro therapy efficacy of the DPP-BDT NPs against HeLa cancer cells was further evaluated. In Fig. 3b, no obvious cytotoxicity of the DPP-BDT NPs was found in the dark. However, the DPP-BDT NPs showed remarkable cytotoxicity upon irradiation with a $660 \mathrm{~nm}$ laser (10 $\mathrm{min}, 0.75 \mathrm{~W} \mathrm{~cm}^{-2}$ ) due to the synergistic cytotoxic effect of PTT and PDT. To further verify the anticancer efficiency of the DPP-BDT NPs, a live/dead cell assay was used to visualize the cell viability, where live and dead cells were stained with calcein AM (green fluorescence) and propidium iodide (PI, red fluorescence), respectively. After incubating HeLa cells with the DPP-BDT NPs in the dark, broad green fluorescence was observed (Fig. 3c), implying negligible dark cytotoxicity of the DPP-BDT NPs.
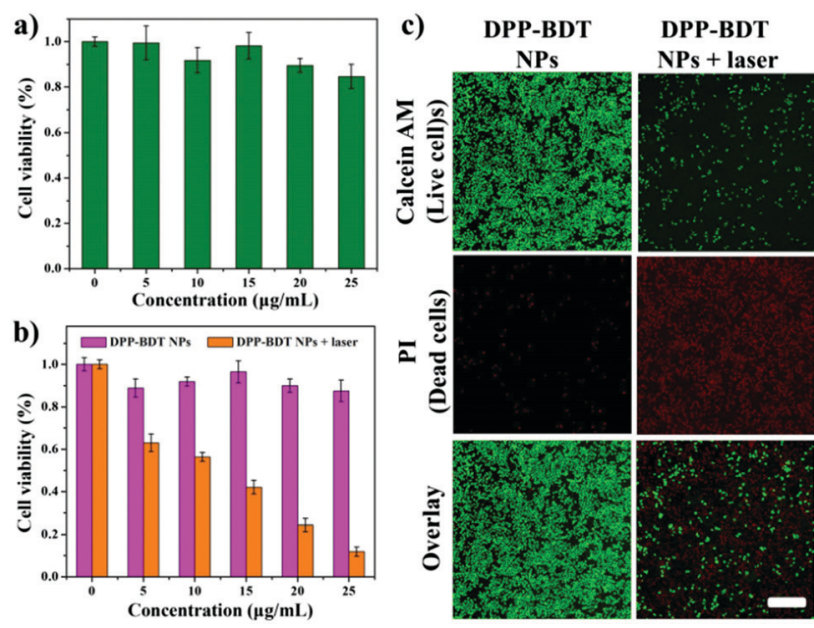

Fig. 3 (a) Biocompatibility assays of DPP-BDT NPs against NIH-3T3 normal cells. (b) Cytotoxicity assays and (c) live/dead assays of HeLa cells treated with DPP-BDT NPs with or without irradiation. Scale bars: $200 \mu \mathrm{m}$. 
a)

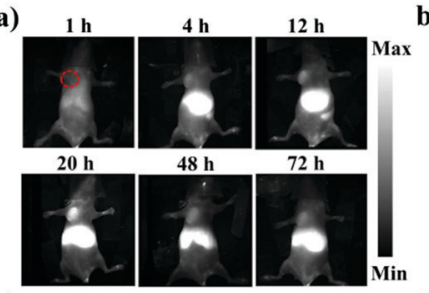

c)

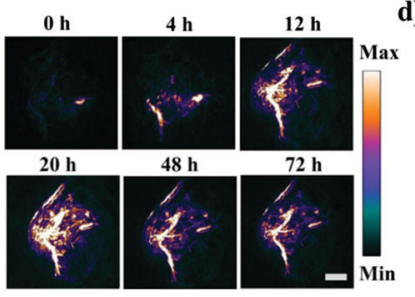

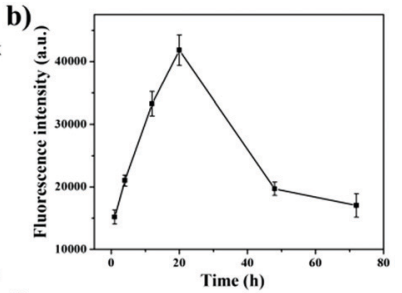

d)

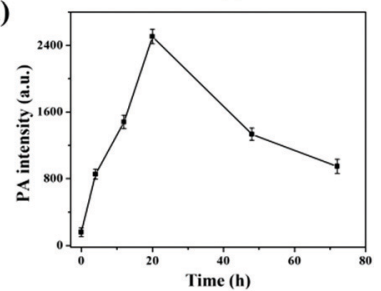

Fig. 4 (a) NIR-II fluorescence imaging, (b) NIR-II signal intensity changes, (c) PA imaging and (d) PA signal intensity changes of HeLa tumor-bearing mice after DPP-BDT NP injection. Scale bars: $1.5 \mathrm{~mm}$.

After laser irradiation, strong red fluorescence was clearly discovered, indicating high light cytotoxicity of the DPP-BDT NPs. Therefore, this outstanding biocompatibility and high light cytotoxicity of the DPP-BDT NPs makes them suitable for cancer therapy.

Next, the NIR-II fluorescence imaging of the DPP-BDT NPs in vivo was further investigated in HeLa tumor-bearing mice. After DPP-BDT NP injection, the tumor region was distinctly imaged with NIR-II fluorescence signals at $4 \mathrm{~h}$ post-injection (Fig. $4 \mathrm{a}$ and b). Meanwhile, the NIR-II signals at the tumor site substantially increased with time and reached a maximum value at $20 \mathrm{~h}$ post-injection, then gradually decreased afterwards. The tumor and major organs of the dissected mice were collected for the biodistribution assay (Fig. S12, ESI $\dagger$ ). The results disclosed that the DPP-BDT NPs were less distributed in the lungs and heart, whereas significant signal intensity in the tumor, kidneys, liver, and spleen was observed, indicating a higher distribution of the DPP-BDT NPs. The PA imaging of the DPP-BDT NPs in vivo was also studied. The PA signal intensity progressively enhanced at $4 \mathrm{~h}$ after DPP-BDT NP injection, and showed a peak value at $20 \mathrm{~h}$ post-injection (Fig. 4c and d). Afterwards, the PA signal intensity decreased gradually over time, which was consistent with the NIR-II fluorescence imaging results. In particular, PA imaging could offer microscopic spatial resolution for tumor diagnosis, which is not likely to be obtained by NIR-II fluorescence imaging, acting as a complementary imaging method compared with NIR-II fluorescence imaging. Therefore, the DPP-BDT NPs, as dual-modal contrast agents for NIR-II fluorescence/PA imaging, could synergistically improve the diagnostic accuracy of tumors.

Subsequently, IR thermal imaging was recorded to confirm the photothermal effect of the DPP-BDT NPs in vivo. Upon $660 \mathrm{~nm}$ laser $\left(1.0 \mathrm{~W} \mathrm{~cm}^{-2}\right)$ irradiation for $10 \mathrm{~min}$ at $20 \mathrm{~h}$ postinjection of the DPP-BDT NPs, a rapid temperature rise could be clearly observed, while an insignificant change in temperature was found in the control group, indicating the excellent photothermal effect of the DPP-BDT NPs (Fig. S13, ESI + ). For the
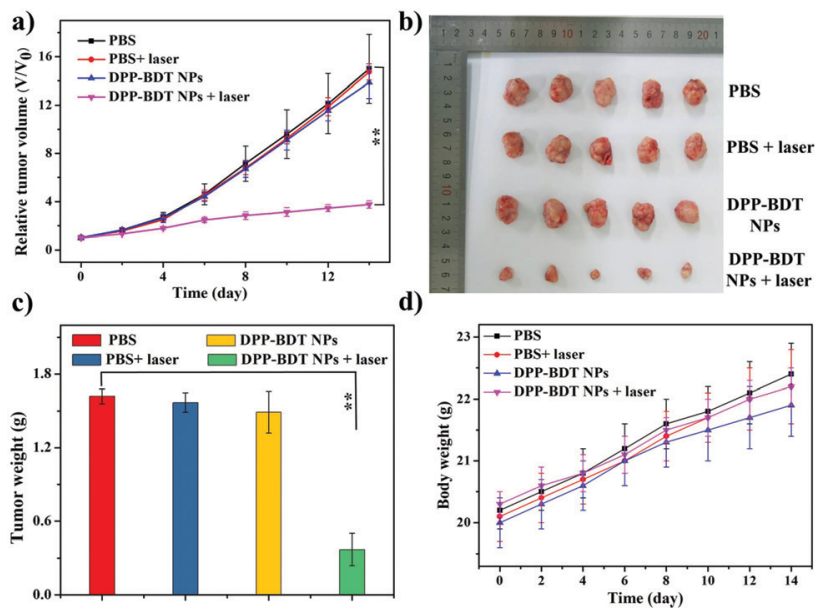

Fig. 5 (a) Tumor growth curves for the various treatment groups $\left({ }^{* *} p<0.01\right)$. (b) Tumor images and (c) tumor weights for the various treatment groups after therapy $\left({ }^{* *} p<0.01\right.$ ). (d) Body weight changes for the various treatment groups.

tumor therapy experiment, mice bearing HeLa tumors were divided into four groups and treated differently: (1) PBS, (2) PBS + laser, (3) DPP-BDT NPs, and (4) DPP-BDT NPs + laser. The combined therapeutic effect of the DPP-BDT NPs was evaluated by monitoring the tumor volume during therapy. The tumor growth rates of both the PBS + laser group and DPP-BDT NPs group were similar to that of the PBS group, confirming no therapeutic effect of both the NIR laser and DPP-BDT NPs (Fig. 5a and b). In contrast, in the DPP-BDT NPs + laser group, tumor growth was significantly suppressed because of the synergetic PTT/PDT effect, which was consistent with the results in vitro. Additionally, no noticeable changes in body weight of the mice were found for all the groups, indicating negligible organism toxicity of the DPP-BDT NPs (Fig. 5d). Moreover, negligible pathological changes of normal major organs were observed for all the groups from the hematoxylin and eosin (H\&E) staining assay, further confirming that the DPP-BDT NPs are innoxious to normal tissues (Fig. 6).

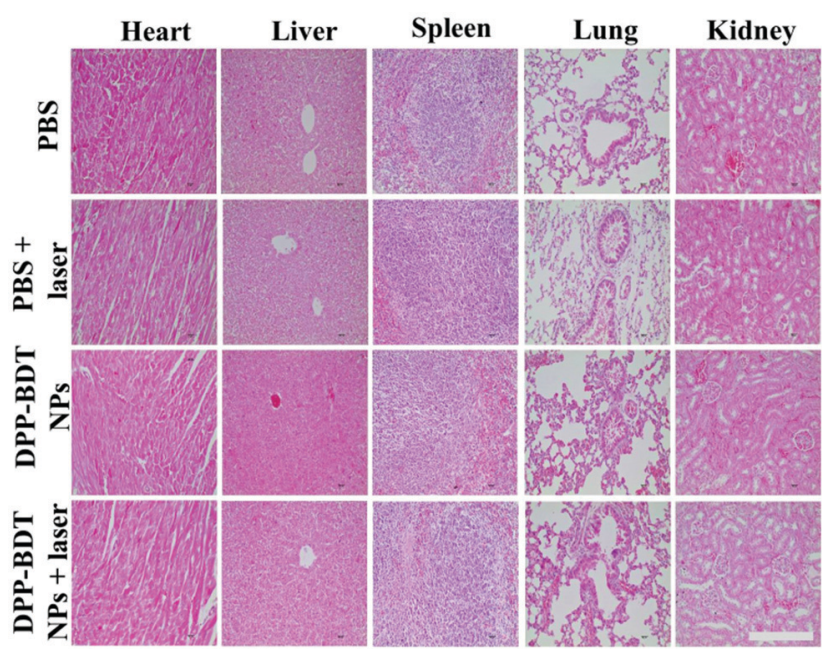

Fig. 6 H\&E staining of major organs. Scale bars: $200 \mu \mathrm{m}$. 


\section{Conclusions}

In conclusion, a novel small-molecule dye, DPP-BDT, with absorption in the NIR-I window and fluorescence emission in the NIR-II region was designed and synthesized, and presented excellent photodynamic and photothermal performance. Multifunctional phototheranostic nanoagents were then successfully developed by encapsulating the small-molecule dye DPP-BDT with amphiphilic DSPE-mPEG5000. From in vitro and in vivo results, such nanoagents showed excellent tumor destruction abilities under a single wavelength of laser irradiation, benefiting from the NIR-II fluorescence/PA dual-modal imaging guided PTT/ PDT combination therapy. This work offers an innovative tactic to fabricate novel phototheranostics for multiple imaging-guided combination therapy, which will open even broader horizons for precise cancer theranostics.

\section{Conflicts of interest}

There are no conflicts to declare.

\section{Acknowledgements}

This work was supported by the National Natural Science Foundation of China (21602112, 21674048, and 21575069), the China Postdoctoral Science Foundation (2017M611876), the 333 project of Jiangsu province (BRA2016379), and the Primary Research \& Development Plan of Jiangsu Province (BE2016770).

\section{Notes and references}

1 (a) G. Feng and B. Liu, Acc. Chem. Res., 2018, 51, 1404-1414; (b) T. Yang, Y. Wang, H. Ke, Q. Wang, X. Lv, H. Wu, Y. Tang, X. Yang, C. Chen, Y. Zhao and H. Chen, Adv. Mater., 2016, 28, 5923-5930; (c) Y. Cai, W. Si, W. Huang, P. Chen, J. Shao and X. Dong, Small, 2018, 14, 1704247; (d) J. Li and K. Pu, Chem. Soc. Rev., 2019, 48, 38-71; (e) Y. Jiang and K. Pu, Acc. Chem. Res., 2018, 51, 1840-1849; $(f)$ X. Ni, X. Zhang, X. Duan, H.-L. Zheng, X.-S. Xue and D. Ding, Nano Lett., 2019, 19, 318-330.

2 (a) Y. Wang, T. Yang, H. Ke, A. Zhu, Y. Wang, J. Wang, J. Shen, G. Liu, C. Chen, Y. Zhao and H. Chen, Adv. Mater., 2015, 27, 3874-3882; (b) H. Chen, W. Zhang, G. Zhu, J. Xie and X. Chen, Nat. Rev. Mater., 2017, 2, 17024; (c) W. Fan, B. Yung, P. Huang and X. Chen, Chem. Rev., 2017, 117, 13566-13638; (d) A. J. Shuhendler, K. Pu, L. Cui, J. P. Uetrecht and J. Rao, Nat. Biotechnol., 2014, 32, 373; (e) H. Gao, X. Zhang, C. Chen, K. Li and D. Ding, Adv. Biosyst., 2018, 2, 1800074.

3 (a) S. He, J. Song, J. Qu and Z. Cheng, Chem. Soc. Rev., 2018, 47, 4258-4278; (b) S. Zhu, B. C. Yung, S. Chandra, G. Niu, A. L. Antaris and X. Chen, Theranostics, 2018, 8, 4141-4151; (c) Y. Kenry and B. Duan, Liu, Adv. Mater., 2018, 30, 1802394; (d) G. Hong, S. Diao, A. L. Antaris and H. Dai, Chem. Rev., 2015, 115, 10816-10906; (e) Y. Jiang, J. Li, X. Zhen, C. Xie and K. Pu, Adv. Mater., 2018, 30, 1705980.

4 (a) G. Hong, J. C. Lee, J. T. Robinson, U. Raaz, L. Xie, N. F. Huang, J. P. Cooke and H. Dai, Nat. Med., 2012, 18,
1841-1846; (b) G. Hong, J. T. Robinson, Y. Zhang, S. Diao, A. L. Antaris, Q. Wang and H. Dai, Angew. Chem., Int. Ed., 2012, 51, 9818-9821; (c) R. Wang, L. Zhou, W. Wang, X. Li and F. Zhang, Nat. Commun., 2017, 8, 14702; (d) Y. Jiang and K. Pu, Adv. Biosyst., 2018, 2, 1700262.

5 (a) Y. Jiang and K. Pu, Small, 2017, 13, 1700710; (b) J. Qi, Y. Fang, R. T. K. Kwok, X. Zhang, X. Hu, J. W. Y. Lam, D. Ding and B. Z. Tang, ACS Nano, 2017, 11, 7177-7188; (c) Q. Miao and K. Pu, Adv. Mater., 2018, 30, 1801778; (d) G. Yu, J. Yang, X. Fu, Z. Wang, L. Shao, Z. Mao, Y. Liu, Z. Yang, F. Zhang, W. Fan, J. Song, Z. Zhou, C. Gao, F. Huang and X. Chen, Mater. Horiz., 2018, 5, 429-435; (e) Y. Jiang, P. K. Upputuri, C. Xie, Y. Lyu, L. Zhang, Q. Xiong, M. Pramanik and K. Pu, Nano Lett., 2017, 17, 4964-4969; $(f)$ J. Qi, C. Chen, X. Zhang, X. Hu, S. Ji, R. T. K. Kwok, J. W. Y. Lam, D. Ding and B. Z. Tang, Nat. Commun., 2018, 9, 1848.

6 Z. Sheng, B. Guo, D. Hu, S. Xu, W. Wu, W. H. Liew, K. Yao, J. Jiang, C. Liu, H. Zheng and B. Liu, Adv. Mater., 2018, 30, 1800766.

7 (a) K. Cheng, H. Chen, C. H. Jenkins, G. Zhang, W. Zhao, Z. Zhang, F. Han, J. Fung, M. Yang, Y. Jiang, L. Xing and Z. Cheng, ACS Nano, 2017, 11, 12276-12291; (b) T. Yang, Y. a. Tang, L. Liu, X. Lv, Q. Wang, H. Ke, Y. Deng, H. Yang, X. Yang, G. Liu, Y. Zhao and H. Chen, ACS Nano, 2017, 11, 1848-1857.

8 (a) H. Gong, Z. Dong, Y. Liu, S. Yin, L. Cheng, W. Xi, J. Xiang, K. Liu, Y. Li and Z. Liu, Adv. Funct. Mater., 2014, 24, 6492-6502; (b) H. Zhu, P. Cheng, P. Chen and K. Pu, Biomater. Sci., 2018, 6, 746-765; (c) Y. Lyu, Y. Fang, Q. Miao, X. Zhen, D. Ding and K. Pu, ACS Nano, 2016, 10, 4472-4481; (d) X. Zhen, J. Zhang, J. Huang, C. Xie, Q. Miao and K. Pu, Angew. Chem., Int. Ed., 2018, 57, 7804-7808.

9 (a) C. Chen, Z. Song, X. Zheng, Z. He, B. Liu, X. Huang, D. Kong, D. Ding and B. Z. Tang, Chem. Sci., 2017, 8, 2191-2198; (b) Z. Yang, Y. Dai, C. Yin, Q. Fan, W. Zhang, J. Song, G. Yu, W. Tang, W. Fan, B. C. Yung, J. Li, X. Li, X. Li, Y. Tang, W. Huang, J. Song and X. Chen, Adv. Mater., 2018, 30, 1707509; (c) J. Mou, T. Lin, F. Huang, H. Chen and J. Shi, Biomaterials, 2016, 84, 13-24; (d) K. Zhang, Z. Yang, X. Meng, Y. Cao, Y. Zhang, W. Dai, H. Lu, Z. Yu, H. Dong and X. Zhang, Mater. Chem. Front., 2018, 2, 1184-1194.

10 (a) W. Tang, Z. Yang, S. Wang, Z. Wang, J. Song, G. Yu, W. Fan, Y. Dai, J. Wang, L. Shan, G. Niu, Q. Fan and X. Chen, ACS Nano, 2018, 12, 2610-2622; (b) C. Liang, S. Diao, C. Wang, H. Gong, T. Liu, G. Hong, X. Shi, H. Dai and Z. Liu, Adv. Mater., 2014, 26, 5646-5652; (c) Q. Wang, P. Zhang, J. Xu, B. Xia, L. Tian, J. Chen, J. Li, F. Lu, Q. Shen, X. Lu, W. Huang and Q. Fan, ACS Appl. Bio Mater., 2018, 1, 70-78; (d) X. Zhen, C. Xie and K. Pu, Angew. Chem., Int. Ed., 2018, 57, 3938-3942.

11 K. Hayashi, M. Nakamura, H. Miki, S. Ozaki, M. Abe, T. Matsumoto, T. Kori and K. Ishimura, Adv. Funct. Mater., 2014, 24, 503-513.

12 (a) Q. Wang, L. Tian, J. Xu, B. Xia, J. Li, F. Lu, X. Lu, W. Wang, W. Huang and Q. Fan, Chem. Commun., 2018, 54, 10328-10331; (b) T. Sun, X. Chen, X. Wang, S. Liu, J. Liu and Z. Xie, Mater. Chem. Front., 2019, 3, 127-136. 
13 (a) Y. Wang, L. Feng and S. Wang, Adv. Funct. Mater., 2018, 1806818; (b) L. Pan, J. Liu and J. Shi, Chem. Soc. Rev., 2018, 47, 6930-6946.

14 (a) F. Hu, C. Li, Y. Zhang, M. Wang, D. Wu and Q. Wang, Nano Res., 2015, 8, 1637-1647; (b) C. Wu, Y. Zhang, Z. Li, C. Li and Q. Wang, Nanoscale, 2016, 8, 12531-12539.

15 Y. Cai, P. Liang, Q. Tang, X. Yang, W. Si, W. Huang, Q. Zhang and X. Dong, ACS Nano, 2017, 11, 1054-1063.

16 (a) A. L. Antaris, H. Chen, K. Cheng, Y. Sun, G. Hong, C. Qu, S. Diao, Z. Deng, X. Hu, B. Zhang, X. Zhang, O. K. Yaghi,
Z. R. Alamparambil, X. Hong, Z. Cheng and H. Dai, Nat. Mater., 2016, 15, 235-242; (b) A. L. Antaris, H. Chen, S. Diao, Z. Ma, Z. Zhang, S. Zhu, J. Wang, A. X. Lozano, Q. Fan, L. Chew, M. Zhu, K. Cheng, X. Hong, H. Dai and Z. Cheng, Nat. Commun., 2017, 8, 15269; (c) J. Qi, C. Sun, A. Zebibula, H. Zhang, R. T. K. Kwok, X. Zhao, W. Xi, J. W. Y. Lam, J. Qian and B. Z. Tang, Adv. Mater., 2018, 30, 1706856; (d) G. Xu, Q. L. Yan, X. G. Lv, Y. Zhu, K. Xin, B. Shi, R. C. Wang, J. Chen, W. Gao, P. Shi, C. H. Fan, C. C. Zhao and H. Tian, Angew. Chem., Int. Ed., 2018, 57, 3626-3630. 